\title{
Formación del capital humano para la salud en Cuba
}

\author{
Juan Vela-Valdés, ${ }^{1}$ Ramón Syr Salas-Perea, ${ }^{1}$ María Luisa Quintana-Galende, ${ }^{1}$ \\ Nayra Pujals-Victoria, ${ }^{1}$ Jorge González-Pérez, ${ }^{2}$ Lázaro Díaz-Hernández, ${ }^{1}$ \\ Lizette Pérez-Perea ${ }^{3}$ y María J.Vidal-Ledo ${ }^{1}$
}

Forma de citar: Vela-Valdés J, Salas-Perea RS, Quintana-Galende ML, Pujals-Victoria N, González Pérez J, Díaz Hernández L, et al. Formación del capital humano para la salud en Cuba. Rev Panam Salud Publica. 2018;42:e33. https://doi.org/10.26633/RPSP.2018.33

RESUMEN Se caracterizan los rasgos distintivos del sistema de formación de los profesionales y los técnicos de la salud en Cuba, y se describen las adecuaciones de los modelos educativos para satisfacer las necesidades de los servicios de salud y su adaptación al entorno socioeconómico y científico-técnico. La educación para la salud es universal, gratuita y está vinculada estrechamente al Sistema Nacional de Salud. Los currículos se enfocan en la interrelación entre la formación académica con la clínica y la actividad comunitaria, cuya forma fundamental de enseñanza-aprendizaje es la educación en el trabajo. El principio fundamental de la educación médica es la integración docente, asistencial e investigativa en el sistema de salud, lo que garantiza que los estudiantes se incorporen a los escenarios docente-asistenciales mediante la interacción de la universidad con la sociedad. Los diseños curriculares están basados en los principales problemas de salud de la población y el medioambiente, con hincapié en la atención primaria de salud. Desde 1959 se han graduado cerca de 350000 profesionales; de ellos, 41000 extranjeros. Además, en 12 países se forman más de 30000 estudiantes con las brigadas médicas internacionalistas cubanas. En la actualidad, existen elevadas matrículas que incrementan la carga docente-asistencial de los profesores. Se necesita fomentar el empleo de docentes y tutores jóvenes y la formación de profesores de ciencias básicas.

Palabras clave Servicios de integración docente asistencial; estudiantes del área de la salud; educación médica; recursos humanos; Cuba.

La política de formación de los profesionales y los técnicos de la salud en Cuba se ha nutrido de diversas influencias: los estudios relacionados con el impacto del modelo educativo recomendado

\footnotetext{
Escuela Nacional de Salud Pública, La Habana, Cuba. Enviar la correspondencia a Juan Vela Valdés, jvela@infomed.sld.cu

2 Dirección Nacional de Docencia Médica, Ministerio de Salud Pública, La Habana, Cuba.

3 Representación de la Organización Panamericana de la Salud, La Habana, Cuba.
}

por Flexner (1) -y su contribución a la consolidación del paradigma biomédico clínico individual y curativo-, el cual se ha mantenido casi inalterable por más de un siglo; la Reforma de Córdoba, Argentina, en 1918 (2), con su marcada proyección social de los diseños curriculares; la Declaración de Alma-Ata, Kazajistán, en 1978 (3); y las reuniones de la Organización de las Naciones Unidas para la Educación, la Ciencia y la Cultura (UNESCO) como la Conferencia Regional de
Educación Superior en América Latina y el Caribe (4); y el pensamiento y la acción de Fidel Castro Ruz. Todas estas contribuciones permitieron perfilar su paradigma: la atención primaria de salud (APS).

Antes de 1959, solo existía una Escuela de Medicina en el país, creada en 1728, y una Escuela de Odontología, fundada en 1900, ambas ubicadas en la capital. El ingreso era limitado por el costo de la matrícula y los altos precios de los libros 
de textos, y los egresados tenían como mercado laboral las capitales provinciales o debían emigrar del país. Los planes de estudios eran teóricos y esencialmente curativos, se enfocaban solo en los aspectos biológicos de la enfermedad y preparaban al profesional para la práctica privada, carente del enfoque social de la salud $(5,6)$.

En 1959 se inició una nueva etapa en la salud pública y en la educación médica universitaria. Aunque en Cuba se constituyó la Secretaría de Sanidad y Beneficencia en una fecha tan temprana como 1909, en 1960 fue necesario reconstruir el actual Ministerio de Salud Pública (MINSAP) para crear un sistema único, estatal y nacional de salud, que definiera las políticas a corto, mediano y largo plazos. En 1962, se proclamó la Ley de Reforma de la Enseñanza Superior, que brindó a los futuros profesionales de la salud la orientación social y humanística que requería el país (7). Cuando en 1982, Fidel Castro Ruz, presidente del Consejo de Estado de la República de Cuba, expresó "La docencia existe para la asistencia...", estaba definiendo el propósito principal de la formación del capital humano en la salud (8).

La formación de los profesionales en las ciencias médicas cubanas tiene un enfoque científico y humanista. Los currículos contemplan las ciencias sociales y las específicas de la salud, el aprendizaje ético en la práctica social, así como el trabajo grupal atendido por tutores (9). En las carreras se promueve una actitud internacionalista y solidaria que se va consolidando en la práctica laboral mediante el aprendizaje y el intercambio entre educandos cubanos y extranjeros. Los egresados están comprometidos moral y científicamente para desempeñarse en Cuba o en otros países a través de la colaboración internacionalista. Otra característica fundamental es la inserción de los estudiantes en los escenarios reales de atención al paciente en las tres instancias de prestación de servicios: comunitaria, hospitalaria y en institutos de investigaciones $(9,10)$.

En este artículo se caracterizan los rasgos distintivos del sistema de formación de los profesionales y los técnicos de la salud en Cuba, y se describen las adecuaciones de los modelos educativos para satisfacer las necesidades de los servicios de salud y su adaptación al entorno socioeconómico y científico-técnico.

\section{FORMACIÓN PROFESIONAL EN LA SALUD}

A partir de 1959 en todas las universidades la matrícula y la adquisición de los libros de textos se tornaron gratuitas, lo que posibilitó progresivamente la masificación del ingreso a estos estudios. Esto condujo a la necesidad de universalizar y extender la formación de los estudiantes de ciencias médicas a todas las provincias del país, por lo que se estableció el plan de becas universitarias.

Para ello fue necesario fomentar de forma paulatina la creación de una red de centros de educación médica superior en el país. Desde 1976, esta red se encuentra subordinada al MINSAP —aunque se rige metodológicamente por el Ministerio de Educación Superior- y se estructura en 13 universidades de ciencias médicas que integran 23 facultades de ciencias médicas, cuatro facultades de estomatología, una facultad de enfermería, una facultad de tecnología de la salud, tres facultades de enfermería y tecnología de la salud, y 15 filiales de ciencias médicas —donde solo se cursan los años clínico-epidemiológicos de cada carrera-, además de dos facultades de ciencias médicas independientes. Existen otras dos universidades: la Escuela Latinoamericana de Medicina, creada en 1999, en la cual se forma gratuitamente a jóvenes de Latinoamérica y el Caribe, África, Asia y los Estados Unidos de América como médicos generales orientados hacia el trabajo en la APS, y la Escuela Nacional de Salud Pública, encargada de la formación y el desarrollo del capital humano en la salud pública en la modalidad de posgrado, como doctorados, especialidades, maestrías y otras formas organizativas de la docencia (11). Cada facultad se estructura, a su vez, en

CUADRO 1. Profesionales de la salud graduados en Cuba, 1959-2016

\begin{tabular}{lc}
\hline \multicolumn{1}{c}{ Carrera } & Graduados \\
\hline Medicina & 158171 \\
Estomatología & 24863 \\
Licenciatura en Enfermería & 82704 \\
Licenciatura en Psicología & 80515 \\
Licenciatura en Tecnología de la Salud & 3008 \\
Total & 349261 \\
\hline
\end{tabular}

Fuente: Elaborado por los autores a partir de referencia 11.

CUADRO 2. Matrícula de estudiantes en las carreras de ciencias médicas. Cuba, curso 2016-2017

\begin{tabular}{|c|c|c|}
\hline Carrera & Número de estudiantes & Años de la carrera \\
\hline \multicolumn{3}{|l|}{ Educación superior } \\
\hline Medicina & $60098^{a}$ & 6 \\
\hline Estomatología & 8289 & 5 \\
\hline Licenciatura en Enfermería & 3763 & 5 \\
\hline Otras licenciaturas ${ }^{b}$ & 4148 & 5 \\
\hline Total & $76298^{c}$ & \\
\hline \multicolumn{3}{|l|}{ Estudiantes extranjeros por carreras (educación superior) } \\
\hline Medicina & 8941 & 6 \\
\hline Estomatología & 44 & 5 \\
\hline Licenciatura en Enfermería & 11 & 5 \\
\hline Licenciatura en Tecnología de la Salud & 286 & 5 \\
\hline Total & 9282 & \\
\hline \multicolumn{3}{|l|}{ Técnicos de la salud } \\
\hline 22 carreras (para graduados de enseñanza media superior) ${ }^{d}$ & $\begin{array}{l}10508 \text { (incluye } 61 \\
\text { estudiantes extranjeros) }\end{array}$ & 2,3 \\
\hline $\begin{array}{l}5 \text { carreras (para graduados de enseñanza media básica) } \\
\text { Total }\end{array}$ & $\begin{array}{l}11617 \\
22125\end{array}$ & 3,3 \\
\hline \multicolumn{3}{|c|}{$\begin{array}{l}\text { a De ellos } 2626 \text { estudian en la Escuela Latinoamericana de Medicina. } \\
\text { 'Otras licenciaturas: Comprende las carreras de Bioanálisis Clínico, Higiene y Epidemiología, Imagenología y Radiofísica } \\
\text { Médica, Logofonoaudiología, Nutrición, Optometría y Óptica, Rehabilitación en Salud, y Sistema de Información en Salud. } \\
\text { c Incluye a los estudiantes extranjeros. } \\
\text { d } 22 \text { carreras: Comprende la formación de técnicos en Enfermería, Análisis Clínicos, Biofísica Médica, Higiene y Epidemiología, } \\
\text { Medicina Transfusional, Podología, Terapia Ocupacional, Vigilancia y Lucha Antivectorial, Citohistotanatología, Inmunoalergia, } \\
\text { Optometría y Óptica, Prótesis Estomatológica, Servicio Farmacéutico, Trabajo Social, Dietética, Fisiología Humana, } \\
\text { Logofonoaudiología, Radiología, Terapia Física y Rehabilitación, Traumatología, Electromedicina y Estadística de Salud. } \\
\text { e } 5 \text { carreras: Abarca la formación de técnicos en Ortoprótesis, Estadística de Salud, Atención Estomatológica, } \\
\text { Electromedicina, y Vigilancia y Lucha Antivectorial. } \\
\text { Fuente: Elaborado por los autores a partir de la referencia } 11 .\end{array}$} \\
\hline
\end{tabular}


departamentos docentes y se anexan a ella instituciones de salud debidamente acreditadas —donde se desarrollan los procesos docentes- compuestas por 451 policlínicos, 10782 consultorios del médico de la familia, 111 clínicas estomatológicas y 150 hospitales (generales, clínico-quirúrgicos, ginecoobstétricos $\mathrm{y}$ pediátricos) (11).

Desde 1959 se han graduado cerca de 350000 profesionales (cuadro 1) y se cuenta con una matrícula de más de 76000 estudiantes de educación superior en el curso 2016-2017 (cuadro 2). Todos los egresados de estas instituciones docentes tienen una ubicación laboral inmediata con su correspondiente contrato de trabajo.

\section{Principios de la formación profesional para la salud en Cuba}

Para entender la formación del capital humano para la salud en Cuba es preciso tener en cuenta las características del Sistema Nacional de Salud (SNS) y sus perfeccionamientos sucesivos. Su principal cambio consistió en la proclamación de la salud como un derecho del pueblo y un deber del Estado; además, la atención de la salud comenzó a tener una orientación preventivo-curativa-rehabilitativa, encaminada hacia la promoción de salud $(12,13)$.

La educación médica cubana ha estado ligada desde 1959 al desarrollo del SNS, y, desde la Declaración de Alma-Ata, se benefició con la concepción de la atención primaria y la medicina familiar (3). Por ello, la formación del capital humano se corresponde con las necesidades del SNS, con hincapié en el fortalecimiento de la medicina comunitaria que se articula con la atención hospitalaria según la estrategia de la APS (12-14).

Entre los principios didácticos que sirven de base a la formación de los profesionales de la salud en Cuba se destacan los siguientes (9):

- Integración docente, asistencial e investigativa en el SNS (que incluye las universidades): las instituciones docentes, asistenciales e investigativas se convierten en "complejos de salud", que son la base de esta integración $(12,15,16)$.

- Carácter científico del proceso docente educativo: es necesaria la actualización científica del proceso docente, en sus aspectos filosófico, temático y pedagógico. Para impartir los contenidos de su disciplina, el profesor debe estar actualizado en los últimos avances de la ciencia y ser capaz de transmitir a los educandos los contenidos esenciales, aunque sin excederse en la información; también debe demostrar el vínculo entre estos con la realidad social y su posible aplicación.

- Enfoque sistémico de las categorías didácticas: el para qué, el qué y el cómo enseñar constituyen una unidad desde el punto de vista didáctico; de esta unidad depende la calidad de la respuesta que la universidad da a las exigencias de la sociedad.

- Vinculación de la teoría con la práctica: se concreta en la combinación del estudio y el trabajo en los servicios de salud y la comunidad.

- Educación en el trabajo: debe entenderse como la formación del educando mediante la práctica en la salud (comunitaria y hospitalaria) a partir de las clases teóricas, lo que le permite profundizar y consolidar los conocimientos, las habilidades intelectuales, los valores y las conductas profesionales en los propios servicios de salud. Es la dimensión académica de los principios de estudio-trabajo y de vinculación teoría-práctica, cuyos espacios de desarrollo son la unidad docente-asistencial y la comunidad $(16,17)$. Ello permite que los procesos formativos se sustenten en los modelos pedagógicos de la actividad (aprender haciendo) y la comunicación (relación profesor-educando y educando-paciente-familia), y se eleve la calidad de la asimilación de los contenidos, pues las actividades formativas están relacionadas con la futura práctica profesional $(16,17)$.

Mientras brinda atención de salud, el profesor contribuye a formar y superar a los educandos, y para ello aplica un pensamiento científico y crítico con el empleo de los métodos profesionales (clínico, epidemiológico, atención de enfermería y tecnológico, según el perfil de cada carrera). La educación en el trabajo, más que una forma de enseñanza, es el principio rector de la educación médica cubana (16).

Cuba ha tenido que enfrentar necesidades y dificultades en la formación del capital humano, entre las que predominan las elevadas matrículas, la sobrecarga de la infraestructura docente-asistencial, el insuficiente completamiento de la plantilla de los profesores de ciencias básicas y la poca preparación pedagógica de docentes y tutores jóvenes (cuadro 3 ). A pesar de ello, es innegable el éxito en la formación de los profesionales de la salud en Cuba, cuyos principales logros se resumen en el cuadro 4 .

\section{CARRERAS DE FORMACIÓN PROFESIONAL}

En las carreras de ciencias médicas se fusionó el modelo pedagógico con el modelo sanitario (promoción, prevención, curación y rehabilitación) y todas mantienen el esquema flexneriano de organización de sus currículos en ciclos: el básico, el básico-clínico, el clínico y la práctica preprofesional; aunque se desarrollan bajo las condiciones históricas particulares de Cuba. Los estudios culminan con la realización de un examen teórico-práctico estatal de alcance nacional $(17,18)$. Aunque en la calidad de los profesionales que se están formando influye la del diseño curricular, lo que la determina realmente es la actitud y el compromiso de los profesores y los educandos. Todos los planes de estudio dirigidos a los profesionales y los técnicos de la salud son únicos, de carácter nacional y de obligatorio cumplimiento en todas las universidades. En su elaboración

\section{CUADRO 3. Principales necesidades y dificultades de la formación del personal para} la salud cubana

- Elevadas matrículas de los estudiantes de pregrado y posgrado que causan una sobrecarga de la infraestructura docente-asistencial

- Incompleta la plantilla de profesores de ciencias básicas

- Alta carga docente y asistencial de los profesores del área clínica y comunitaria

- La preparación pedagógica de docentes y tutores jóvenes no alcanza los objetivos propuestos

- No se ha logrado el óptimo aprovechamiento del tiempo por parte de los estudiantes vinculados a la educación en el trabajo

- Insuficiente tiempo dedicado al estudio, falta de métodos de estudio adecuados y aún limitado uso de la literatura docente

- El número de computadoras y simuladores no satisface las necesidades docentes de tan alta matrícula - Deficiencias en el proceso de evaluación del aprendizaje

Fuente: Elaborado por los autores. 
CUADRO 4. Principales logros en la enseñanza médica cubana mediante la educación en el trabajo

- Se ha desarrollado en los profesores una visión integral de los procesos formativos y se ha estimulado la creación de recursos didácticos específicos para cada unidad curricular.

- Se ha propiciado una mayor calidad en la explicación científica de hipótesis y teorías.

- Se ha incentivado la relación del estudiante con los pacientes y sus familiares en las distintas modalidades académicas.

- La educación en el trabajo ha permitido comprender que la ética en la salud no solo se aprende en las aulas, sino mediante el trabajo en el servicio de salud y la comunidad; esto ha posibilitado que los educandos se formen en el marco de los procesos laborales, aprendan y consoliden los valores humanos y la ética laboral.

- Se ha logrado que los educandos mantengan una correcta conducta profesional, moral, humana y solidaria, y que sientan la necesidad de superarse durante toda su vida laboral.

- Se ha desarrollado en los educandos competencias que concretan mediante los diversos modos de actuación profesional.

- El estudiante se ha apropiado de los métodos de trabajo del profesional de la salud y logra familiarizarse con las tecnologías propias de cada nivel de atención de la salud.

- Se ha logrado que el educando desarrolle un pensamiento crítico-reflexivo, creador e independiente, y aprenda a trabajar en equipos multidisciplinarios.

- El alumno ha aprendido a identificar sus problemas cognoscitivos y a aplicar los métodos científicos en la práctica profesional.

Fuente: Elaborado por los autores.

participan los profesores más destacados de esas instituciones.

Pero la calidad docente depende también de la calidad del claustro en su conjunto. El Ministerio de Educación Superior ha establecido en Cuba tres categorías docentes principales (19): profesor asistente, profesor auxiliar y profesor titular. Existe además la categoría transitoria de instructor, para quienes inician su labor docente. Asimismo, se ha garantizado un sistema para la formación y la superación profesoral -estructurado en curso básico, diplomado, maestría y doctorado- que garantiza el desarrollo científico-pedagógico (9).

Además de la docencia y la asistencia médica, también se estimula la investigación como parte de la formación profesional. La investigación estudiantil se estructura en dos vertientes: 1) como colaboradores en las investigaciones de los residentes, los especialistas y el personal docente; y 2) a través de la actividad científica estudiantil (extracurricular), mediante la cual los educandos se entrenan en la aplicación del método científico en la solución de los problemas de salud y educacionales. Para ello, los estudiantes cuentan con la tutoría de los docentes y presentan sus trabajos en los foros científicos estudiantiles que se realizan anualmente a nivel de facultad, universidades y nacional. Los mejores trabajos se publican en la Revista científico-estudiantil de ciencias médicas 16 de Abril, creada en 1961.

\section{Carrera de Medicina}

El currículo de esta carrera parte de los problemas de salud de la población y prevé la formación de un médico general con aptitudes y actitudes humanistas, y un enfoque hacia la salud más que hacia la enfermedad, y caracterizado por competencias diagnósticas, terapéuticas y comunicativas desarrolladas a partir de la solución de problemas. La carrera tiene como eje la salud del individuo, la familia, la comunidad y el medioambiente. Se encuentra estructurada en seis años y se enfoca en la estrategia de la APS. El educando analiza cada problema de salud, individual o colectivo, desde el punto de vista biopsicosocial, y conjuga lo humanístico y lo ético con lo científico y lo tecnológico (17).

Los planes de estudio se han perfeccionado en la misma medida que han cambiado las características y las demandas del SNS y los compromisos internacionales (18). Su diseño se caracteriza por la responsabilidad social, el pensamiento crítico, la flexibilidad y el intercambio académico, así como por el principio de educación continua a lo largo de toda la vida profesional. Desde el primer año de la carrera, el estudiante se vincula con los procesos de salud y enfermedad a nivel comunitario, mediante una asignatura rectora que se imparte en todos los años: Medicina General Integral.

El proceso formativo está basado en la concepción de aprender a aprender y la creatividad, como ejes de los cambios y las transformaciones requeridos, articulados con el compromiso social y la formación ciudadana del estudiante $(9,17)$. El egresado puede laborar en cualquiera de las unidades del SNS, donde podrá ejercer funciones asistenciales, docentes, investigativas, de gestión de la salud pública u otras más específicas, como es el caso de la medicina en situaciones de desastre.

\section{Carrera de Estomatología}

En 1963 se creó esta carrera en la entonces provincia de Oriente (que ahora comprende las provincias de Granma, Guantánamo, Holguín, Las Tunas y Santiago de Cuba), en 1975 en Las Villas (actualmente las provincias de Cienfuegos, Sancti Spíritus y Villa Clara), y en 1976 en Camagüey (que ahora corresponde a las provincias de Camagüey y Ciego de Ávila). Hoy existen facultades con la carrera de estomatología en las 15 provincias del país $(20,21)$.

Los nuevos conceptos y las necesidades de atención bucal de la población impulsaron al MINSAP a implementar una política estomatológica orientada hacia la salud y no hacia la enfermedad. Para ello se rediseñaron los planes de estudios a fin de preparar estomatólogos generales capaces de alcanzar los objetivos propuestos, en la medida que el cuadro de salud bucal y el modelo de atención estomatológica cambiaba bajo la estrategia de la APS $(22,23)$. En el año 2011 se puso en vigor el plan de estudios actual, organizado en un currículo base con los contenidos que garantizan la formación del profesional y un currículo específico, en el que se efectúa la educación en el trabajo, y se adecua a las características de los territorios y las instituciones. Este plan contempla, además, el diseño de asignaturas optativas en cada facultad (23).

\section{Licenciatura en Enfermería}

En 1976 se inició esta carrera con cuatro años de duración para la formación del personal de enfermería en la modalidad presencial (mediante cursos regulares diurnos); por primera vez, el método de trabajo de estos profesionales se enfocó en el proceso de atención de enfermería (24).

Actualmente la carrera se imparte mediante dos tipos de cursos:

Curso regular diurno de cinco años: se inició en 1987 en La Habana, con un modelo formativo diurno y presencial. A partir de 1988, la carrera se fue extendiendo progresivamente al resto del país (25). El requisito de ingreso es ser graduado de bachillerato; no se exigen estudios previos de enfermería. La forma fundamental de organización docente es 
la educación en el trabajo y el último año de estudios se dedica a la práctica preprofesional a tiempo completo en escenarios laborales.

Curso por encuentros: también con una duración de cinco años, se destina a los trabajadores graduados de nivel técnico en enfermería. En esta modalidad, el estudiante participa en encuentros presenciales con una frecuencia de 3 a 5 días mensuales, en los que recibe las clases y desarrolla las actividades prácticas, aunque sin dejar de laborar como técnico en enfermería en su institución mientras cursa sus estudios. El último semestre permanece a tiempo completo en los escenarios docente-asistenciales del SNS (26).

\section{Licenciatura en Tecnología de la Salud}

La incorporación de equipos modernos en las instituciones hospitalarias y comunitarias ha requerido formar profesionales con una mayor preparación científica y tecnológica, capacitado para el manejo de equipos de alta tecnología para usos diagnóstico y terapéutico. Por eso, en 1989 se creó en La Habana la carrera de Licenciatura en Tecnología de la Salud. Entonces, esta licenciatura tenía seis perfiles de formación y se impartía mediante cursos por encuentros presenciales de una semana al mes. En el 2003 se actualizó el programa curricular y se extendió a 21 perfiles de formación (27). Desde su primera versión, esta carrera desarrolla en los educandos el método tecnológico como objetivo profesional de trabajo.

Sin embargo, la ejecución de esta carrera presentó obstáculos por la diversidad de perfiles sin puntos de contacto; además, no se lograba la preparación de un profesional de perfil amplio ni se cumplía la interrelación entre las disciplinas básicas que se impartían de forma centralizada con las específicas, y el eje de formación común se conseguía solo en las disciplinas generales. Por lo tanto, estos perfiles resultaron estrechos e insuficientes para dar respuesta al desarrollo tecnológico actual (27).

En consecuencia, fue necesario diseñar ocho carreras que se implementaron nacionalmente en el curso 2010-2011, en la modalidad de cursos regulares diurnos con una duración de 5 años (Bioanálisis Clínico, Higiene y Epidemiología, Imagenología y Radiofísica Médica, Logofonoaudiología, Nutrición, Optometría y
Óptica, Rehabilitación en Salud, y Sistema de Información en Salud). En ellas se adquieren los conocimientos y las habilidades prácticas mediante horas dedicadas a la educación en el trabajo. También existe la modalidad de cursos por encuentros, que se imparte a técnicos que se mantienen en sus labores.

\section{FORMACIÓN DE ESTUDIANTES EXTRANJEROS}

Cuba no solo ha formado profesionales de la salud para el país, sino también para el mundo, lo que constituye uno de los programas más humanos y solidarios reconocidos internacionalmente. Hasta el año 2015 se habían graduado más de 41000 profesionales de 121 países; de ellos, 24402 son egresados de 10 graduaciones de la Escuela Latinoamericana de Medicina $(11,28)$ en el curso académico 2016-2017 en Cuba estaban matriculados 9282 estudiantes extranjeros en las carreras de la salud (cuadro 2).

Además de los programas de formación para extranjeros que se ejecutan en Cuba, desde 1976 se ha trabajado en 12 países, en los que se han formado 31117 profesionales; de ellos, 28762 corresponden a la formación de pregrado y 2355 a la de posgrado. Según la información ofrecida por la Dirección Nacional de Docencia del MINSAP, actualmente trabajan 1787 profesores y docentes cubanos en las brigadas médicas de la Isla en 14 países.

Como complemento, los profesores cubanos han colaborado en la creación y el desarrollo de varias facultades de ciencias médicas en otros países. Entre estas se encuentran las de Angola, Eritrea, Etiopía, Gambia, Ghana, Guinea-Bissau, Guinea Ecuatorial, Guyana, Haití, Timor Leste, Uganda, Venezuela y Yemen. En el caso de la República Bolivariana de Venezuela, se debe destacar que se han formado más de 20000 médicos integrales comunitarios (29).

\section{FORMACIÓN DE TÉCNICOS DE LA SALUD}

Como ya se mencionó, a partir de 1959 se estableció una política encaminada a la formación acelerada de profesionales de enfermería y otros técnicos de la salud, y se inició la descentralización de la docencia hacia las provincias, a fin de lograr la preparación del personal necesario en el mismo lugar donde iban a laborar. La educación de técnicos de la salud siguió los mismos principios de vincular el estudio con el trabajo y de integrar la actividad docente con la asistencial.

Así, en la década de 1990 las universidades de ciencias médicas asumieron la formación técnica de estos educandos y se construyeron institutos politécnicos de la salud (27).

Los estudios de enfermería de nivel técnico datan de 1902 y fue la única variante de formación en la especialidad hasta 1976. Las carreras actuales para los alumnos que terminan la enseñanza media superior o bachillerato (12 grados) tienen un plan de 2 años y 15 semanas de duración, mientras que los que terminan la enseñanza media básica (9 grados) deben vencer un plan de 3 años y 15 semanas. En ambos casos, la práctica preprofesional se realiza en los servicios donde serán ubicados posteriormente. Todos los egresados tienen un contrato de trabajo al concluir los estudios (26).

En la actualidad se ofrecen 22 carreras técnicas para alumnos egresados de enseñanza media superior y 5 carreras para los egresados de enseñanza media básica. El plan de estudios de las especialidades técnicas tiene un enfoque integral en el que predominan los contenidos prácticos. El segundo semestre del año terminal se dedica íntegramente a la práctica preprofesional (26).

\section{EDUCACIÓN DE POSGRADO}

En 1962, el MINSAP comenzó la formación de especialistas mediante residencias médicas y estomatológicas, que satisfizo las necesidades de esos momentos. Más tarde, a partir de 1987, en Cuba se implementó un sistema de educación posgraduada, cuya dirección pasó a ser responsabilidad de las universidades de ciencias médicas. El 71,9\% de los médicos graduados en el país durante el período 1962-2013 realizó sus estudios de especialidad en el marco del sistema de residencias en las provincias donde radicaban (15).

En la actualidad existen 63 programas de especialidades médicas y estomatológicas, entre los que se destacan los de Medicina General Integral y Estomatología General Integral por su masividad y el papel que desempeñan en el SNS. El proceso formativo de estas especialidades es un continuum del pregrado y se desarrolla totalmente mediante la educación en el trabajo dentro de los servicios de salud y la comunidad; además, 


\section{CUADRO 5. Lecciones aprendidas}

- Los procesos formativos de los profesionales y los técnicos de la salud deben estar en correspondencia con el encargo social y las necesidades de la población, y no con los intereses propios de cada universidad.

- La docencia existe para la asistencia; el trabajo de profesores y educandos en los servicios de salud y la comunidad incrementa la calidad de la atención de salud de la población.

- La reformulación de los currículos tiene que contemplar los modelos pedagógicos y sanitarios; los planes de estudio deben basarse más en la salud, sin descuidar los problemas que debe ser capaz de resolver el profesional al egresar, y deben abarcar la atención comunitaria bajo la estrategia de la APS.

- Los currículos se deben enfocar en el aprendizaje, y la identificación y la solución de los problemas de salud de las personas, las familias, la comunidad y el medioambiente, y deberán considerar la labor ante los desastres naturales y la colaboración internacional.

- La calidad de los profesionales en formación depende más de la actitud y los compromisos del binomio profesor-educando que de la calidad del diseño curricular.

- Se requiere garantizar la formación y la superación sistemática del cuerpo docente en las ciencias de la educación para la salud.

- La clave para el éxito del proceso docente-educativo para la salud consiste en la integración —o al menos la coordinación - docente, asistencial e investigativa (universidad-salud-sociedad); el carácter científico y sistémico del proceso didáctico; y la educación en el trabajo en las instituciones docente-asistenciales y la comunidad (aprender haciendo).

- En todo proceso formativo para la salud, es esencial que se asegure la formación ética, moral, solidaria, humanística y ciudadana de los educandos, a partir del ejemplo del cuerpo de profesores.

\section{Fuente: Elaborado por los autores.}

se caracteriza por la independencia cognoscitiva en el aprendizaje, con el apoyo de un tutor. Entre 1962 y el 2016 se graduaron 105419 especialistas médicos y estomatólogos (11).

Desde 1976, las maestrías y los doctorados forman parte de la enseñanza de posgrado. Las maestrías se generan por iniciativa de las universidades y deben satisfacer las necesidades objetivas de la educación o del SNS; hoy se ejecutan 56 programas de maestrías. En cambio, los estudios de doctorado en ciencias no han logrado el avance que requiere el SNS por ser insuficiente la cifra de doctores formados (9).

Para garantizar la calidad de los egresados, el posgrado académico necesita de servicios de salud de alta calidad, en los que se capaciten los especialistas. Por ello, la superación profesional de los egresados universitarios y técnicos de la salud es gratuita. Esto posibilita la actualización científico-técnica sistemática para un mejor desempeño de funciones y responsabilidades laborales de los profesionales de la salud (30). En el curso 2014-2015 se realizaron 49665 actividades de superación profesional dentro del SNS, en las que participaron 295819 profesionales de la salud (31).

En el cuadro 5 se exponen las principales lecciones aprendidas en la formación del capital humano para la salud en Cuba.

\section{CONCLUSIONES}

La política educacional cubana en la formación del capital humano para la salud ha estado dirigida a satisfacer las necesidades de la población y del SNS. La formación de los estudiantes es integral y humanista, y se ejecuta esencialmente en los servicios de salud y en la comunidad. A todos los egresados se les garantiza su contrato laboral.

Los currículos se diseñan a partir de los problemas de salud de la población y se estructuran según la estrategia de la APS, que se ha ido perfeccionando sistemáticamente. El proceso docente se enfoca en la integración docente-asistencial-investigativa, la utilización del método propio de cada profesión, la educación en el trabajo y la interacción de la universidad médica con la comunidad. Las elevadas matrículas actuales han complejizado esta integración en los escenarios docente-asistenciales

Desde 1959 se han graduado cerca de 350000 profesionales; de ellos, más de 41000 extranjeros. Además, en 12 países se han formado más de 30000 estudiantes con las brigadas médicas internacionalistas cubanas.

La constante superación profesoral ha caracterizado al claustro de ciencias médicas. Sin embargo, hoy los profesores tienen una elevada carga docente-asistencial, lo que ocasiona nuevos retos para mantener la calidad del claustro y aspirar a estadios superiores.

\section{RECOMENDACIONES}

Se debe mantener la política educacional establecida y perfeccionar la integración de la universidad con los servicios de salud y la comunidad. Ante el aumento de la matrícula, se hace imprescindible el cumplimiento estricto de los currículos. Es necesario incrementar la formación de docentes para ciencias básicas, fomentar el empleo de docentes y tutores jóvenes bien preparados, aumentar la cifra de doctores en ciencias y fortalecer la superación sistemática e integral de los profesores.

\section{Conflicto de intereses. Ninguno.}

Declaración. Las opiniones expresadas en este manuscrito son responsabilidad de los autores y no reflejan necesariamente los criterios ni la política de la Revista Panamericana de Salud Pública / Pan American Journal of Public Health o de la Organización Panamericana de la Salud.

\section{REFERENCIAS}

1. Flexner A. Medical education in the United States and Canada. A report to The Carnegie Foundation for the Advancement of Teaching (with an introduction by Henry S Pritchett, President of the Foundation). Boston, Massachusetts: Updyke; 1910. (Bulletin No. 4).

2. Martín Sabina E. La Reforma de Córdoba. Impactos y continuidad en las experiencias de la República de Cuba. En: Sader E, Gentili P, Aboites H. La reforma universitaria: desafíos y perspectivas noventa años después. Buenos Aires: Consejo Latinoamericano de Ciencias Sociales; 2008.

3. Organización Mundial de la Salud. Formulación de estrategias con el fin de alcanzar Salud para Todos en el año 2000. Principios básicos y cuestiones esenciales. Ginebra: OMS; 1979.

4. Organización de las Naciones Unidas para la Educación, la Ciencia y la Cultura. Conferencia Regional de Educación Superior en América Latina y el Caribe. Declaración Final. Cartagena de Indias:
UNESCO-Instituto Internacional de Educación Superior en América Latina y el Caribe; 2008.

5. Delgado García G. Desarrollo histórico de la enseñanza médica superior en Cuba desde sus orígenes hasta nuestros días. Educ Med Super. 2004;18(1). Disponible en: http://scielo.sld.cu/scielo.php?script=sci arttext\&pid=S0864-21412004000100007\& lng=es Acceso el 23 de abril del 2017.

6. Álvarez Sintes R, Barcos Pina I. La formación y el perfeccionamiento de recursos 
humanos en el sistema de salud cubano. Rev Habanera Cienc Med. 2014;13(3): 368-70. Disponible en: http://scielo.sld. $\mathrm{cu} /$ scielo.php? script $=$ sci_arttext\&pid $=$ S1729-519X2014000300001\&lng=es Acceso el 29 de agosto del 2017.

7. Cuba, Consejo Superior de Universidades. La reforma de la enseñanza superior en Cuba. La Habana: Ministerio de Educación; 1962.

8. Castro Ruz F. Discurso pronunciado en el claustro extraordinario de profesores de ciencias médicas de La Habana, 11 de junio de 1982. La Habana: Editora Política; 1982.

9. Salas Perea RS, Salas Mainegra A. Modelo formativo del médico cubano. Bases teóricas y metodológicas. Capítulo 7. La Habana: Editorial Ciencias Médicas; 2017. Disponible en: http://www.bvs.sld.cu/libros_texto/ modelo_formativo_medico_cubano/cap_7. pdf Acceso el 10 abril del 2017.

10. Fernández Sacasas J. El principio rector de la educación médica cubana. Un reconocimiento a la doctrina pedagógica planteada por Fidel Ilizástigui Dupuy. Educ Med Super. 2013;27(2). Disponible en: www.ems. sld.cu/index.php/ems/article/view/170 Acceso el 10 abril del 2017.

11. Cuba, Ministerio de Salud Pública. Anuario Estadístico de Salud 2016. La Habana: MINSAP; 2017. Disponible en: http:// www.one.cu/aec2016.htm Acceso el 14 de diciembre del 2017.

12. Arteaga Herrera JJ, Chávez Lazo E. Integración docente-asistencial investigativa (IDAI). Educ Med Super. 2000;14(2):1. http:/ / scielo.sld.cu/scielo.php?script=sci_ arttext\&pid=S0864-21412000000200008 Acceso el 10 abril del 2017.

13. Alemañy E, Díaz-Perera G. Medicina familiar en Cuba. Medwave. 2013;13(3):e5634. Disponible en: https://www.medwave. cl/link.cgi / Medwave / Enfoques / SaludFamiliar/5634 Acceso el 14 de diciembre del 2017.

14. Ilizástigui Dupuy F, Douglas Pedroso R. La formación del médico general básico en Cuba. Educ Med Salud. 1993;27(2):189-205.

15. Vela Valdés J. Formación de médicos para los servicios de salud en Cuba 1959-2014. [Tesis doctoral]. La Habana: Escuela Nacional de Salud Pública; 2016. Disponible en: http://www.revsaludpublica.sld.cu/ index.php/spu/article/view/1008/876 Acceso el 15 de septiembre del 2017.

16. Salas Perea RS, Salas Mainegra A. Educación médica contemporánea. Retos, procesos y metodologías. Bucaramanga, Colombia: Universidad de Santander; 2014.

17. Salas Perea RS, Salas Mainegra A. La educación médica cubana. Su estado actual. Rev Docencia Univ. 2012;10:293-326. Disponible en: http://redaberta.usc.es/redu Acceso el 10 agosto del 2017.

18. Vela Valdés J, Salas Perea RS, Pujals Victoria N, Quintana Galende ML, Pérez Hoz G. Planes de estudio de Medicina en Cuba de 1959 a 2010. Educ Med Super. 2016;30(1). Disponible en: http://scielo. sld.cu/scielo.php?script=sci_arttext\&pid$=$ S0864-21412016000100002\&lng $=$ es Acceso el 16 de septiembre del 2017.

19. Cuba, Ministerio de Educación Superior. Resolución Ministerial No. 85/2016 del 17 de octubre del 2016. Reglamento para la aplicación de las categorías docentes de la educación superior. Gac Of Repub Cuba. 2017;5. Disponible en: https:/ / www.gacetaoficial.gob.cu/pdf/GOC-2017-O5.rar Acceso el 14 de diciembre del 2017.

20. Delgado Correa W. Fundación de la Facultad de Medicina de Santiago de Cuba. MEDISAN. 2016;20(3):421-5. Disponible en: http://scielo.sld.cu/scielo.php?script=sci arttext\&pid $=$ S1029-30192016000300020 Acceso el 25 de noviembre del 2016.

21. Companioni F. Contribución a la historia de la estomatología cubana. La Habana: Editorial Ciencias Médicas; 2010. Disponible en: http://gsdl.bvs.sld.cu/PDFs/Coleccion_ Estomatologia/contribucion_estomatologia/contribucion.pdf Acceso el 30 de agosto del 2017.

22. Barciela González-Longoria MC, Grau León IB, Urbizo Vélez J, Soto Cantero LA, Sosa Rosales M. Formación de recursos humanos en estomatología, su impacto en la salud bucal de la población. Educ Med Super. 2017;31(2). Disponible en: http:// www.ems.sld.cu/index.php/ems/article/ view /1132 Acceso el 30 de agosto del 2017.

23. González Naya G, Montero del Castillo M. Estomatología General Integral. La Habana: Editorial Ciencias Médicas; 2013. Disponible en: http://gsdl.bvs.sld.cu/PDFs/Coleccion_ Estomatologia/estomatologia_general_ int/estomatologia_general_completo.pdf Acceso el 10 de septiembre del 2017.

24. Pernas Gómez M. Modelo curricular para la formación de licenciados en enfermería. Cuba 2004-2009. [Tesis doctoral]. La Habana: Universidad de Ciencias Médicas de La Habana; 2013. Disponible en: http:/ / tesis.repo.sld.cu/679/1/Marta_Pernas_
Tesis_doctoral_COMPLETO_sin_paginaci\%C3\%B3n..pdf Acceso el 3 de septiembre del 2017.

25. Pernas Gómez M, Zubizarreta Estévez M, Miralles Aguilera E, Sierra Figueredo S, Diego Cobelo JM, Fernández Sacasas JA. Bases teórico-metodológicas de un modelo curricular pertinente para la formación de licenciados en enfermería en Cuba. Educ Med Super. 2011;25(3):283-300. Disponible en: http://scielo.sld.cu/pdf/ems/v25n3/ ems06311.pdf Acceso el 30 de agosto del 2017.

26. Cuba, Ministerio de Salud Pública. Informe de la Dirección Nacional de Docencia Médica. La Habana: MINSAP; 2017.

27. Rodríguez Gallo MN, García Linares G, García González M, Ortega González N, Sánchez Fernández O. Desarrollo de la formación de técnicos y tecnólogos de la Salud en Cuba. Rev Hum Med. 2011;11(3):489-503. Disponible en: http://scielo.sld.cu/scielo. php script $=$ sci_arttext $\&$ pid $=$ S1727 2011000300006 Acceso el 30 de agosto del 2017.

28. Morales Ojeda R. Cobertura universal de salud. Experiencia cubana. Convención Internacional de Salud Pública Cuba-Salud 2015; La Habana; 2015 abril 20-24. La Habana: Editorial Ciencias Médicas; 2015.

29. Cuba, Ministerio de Salud Pública. Registros administrativos, colaboración internacional. La Habana: MINSAP; 2016.

30. Borges Oquendo LC, Añorga Morales JA. Dimensiones de la evaluación de impacto del posgrado académico desde la óptica de la educación avanzada en la educación médica. Educ Med Super. 2015;29(2). Disponible en: http://scielo.sld.cu/scielo. php ? script $=$ sci_arttext $\&$ pid $=S 0864$ $21412015000200015 \& \operatorname{lng}=e s$ Acceso el 15 de septiembre del 2017.

31. Urbina Laza Omayda. La educación de posgrado en las universidades médicas cubanas. Educ Med Super. 2015;29(2). Disponible en: http://scielo.sld.cu/scielo. php? script $=$ sci_arttext $\&$ pid $=$ S0864 21412015000200017\&lng=es Acceso el 1 de septiembre del 2017.

Manuscrito recibido el 28 de abril de 2017. Aceptado para publicación, tras revisión, el 30 de noviembre del 2017. 
ABSTRACT

\section{Training of human capital for health in Cuba}

Keywords
The distinctive features of the training system of health professionals and technicians in Cuba are characterized, and the adaptations of the educational models to meet the needs of health services and their adaptation to the socio-economic and scientific-technical environment are described. Education for health is universal, free and is closely linked to the National Health System. The curricula focus on the interrelation between academic training and clinical practice and community activity, whose fundamental form of teaching-learning is based on education at work. The fundamental principle of medical education is the integration of teaching, health care, and research within the health system, which ensures that students are embedded into the teaching-care scenarios through the interaction of the university with society. The curricular plans are based on the main health problems of the population and the environment, with emphasis on primary health care. Since 1959, nearly 350000 professionals have graduated, including 41000 foreign students. In addition, more than 30000 students are trained in 12 countries with the Cuban international medical brigades. Currently, high enrollments increase the teaching-care burden of faculty staff and faculty is subject to frequent changes, representing a challenge to maintaining high-quality training. The scarcity of basic science teachers also affects training. The recruitment of teachers and young tutors needs to be encouraged.

Teaching care integration services; students, health occupations; education, medical; human resources; Cuba.
RESUMO

\section{Formação de capital humano para a saúde em Cuba}

Palavras-chave
As características distintivas do sistema de treinamento de profissionais e técnicos de saúde em Cuba são caracterizadas e as adaptações dos modelos educacionais para atender às necessidades dos serviços de saúde e sua adaptação ao ambiente sócio-econômico e científico-técnico são descritas. A educação para a saúde é universal, gratuita e está intimamente ligada ao Sistema Nacional de Saúde. Os currículos centram-se na inter-relação entre o treinamento acadêmico com a prática clínica e a atividade comunitária, cuja forma fundamental de ensino-aprendizagem é a educação no trabalho. O princípio fundamental da educação médica é a integração do ensino, do cuidado de saúde e da pesquisa no sistema de saúde, o que garante que os alunos sejam incorporados nos cenários de ensino-cuidado através da interação da universidade com a sociedade. Os projetos curriculares são baseados nos principais problemas de saúde da população e do meio ambiente, com ênfase na atenção primária à saúde. Desde 1959, cerca de 350000 profissionais se formaram, incluindo 41000 profissionais estrangeiros. Além disso, mais de 30000 alunos são treinados em 12 países com as brigadas médicas internacionalistas cubanas. Atualmente, há altas matrículas que aumentam a carga de ensino-cuidado dos professores e mudanças freqüentes são feitas na faculdade, o que complicou os desafios para manter um treinamento de alta qualidade. A escassez de professores de ciências básicas também afeta o treinamento. O emprego de professores e jovens tutores deve ser encorajado.

Serviços de integração docente-assistencial; estudantes de ciências da saúde; educação médica; recursos humanos; Cuba. 